\title{
Comparative effect of angiotensin II type I receptor blockers and calcium channel blockers on laboratory parameters in hypertensive patients with type 2 diabetes
}

Yayoi Nishida ${ }^{1,2}$, Yasuo Takahashi ${ }^{1,2^{*}}$, Tomohiro Nakayama ${ }^{4}$ and Satoshi Asai ${ }^{2,3}$

\begin{abstract}
Background: Both angiotensin I| type I receptor blockers (ARBs) and calcium channel blockers (CCBs) are widely used antihypertensive drugs. Many clinical studies have demonstrated and compared the organ-protection effects and adverse events of these drugs. However, few large-scale studies have focused on the effect of these drugs as monotherapy on laboratory parameters. We evaluated and compared the effects of ARB and CCB monotherapy on clinical laboratory parameters in patients with concomitant hypertension and type 2 diabetes mellitus.
\end{abstract}

Methods: We used data from the Clinical Data Warehouse of Nihon University School of Medicine obtained between Nov 1, 2004 and July 31, 2011, to identify cohorts of new ARB users ( $n=601)$ and propensity-score matched new CCB users $(n=601)$, with concomitant mild to moderate hypertension and type 2 diabetes mellitus. We used a multivariate-adjusted regression model to adjust for differences between ARB and CCB users, and compared laboratory parameters including serum levels of triglyceride (TG), total cholesterol (TC), non-fasting blood glucose, hemoglobin $\mathrm{A} 1 \mathrm{c}(\mathrm{HbA} 1 \mathrm{c})$, sodium, potassium, creatinine, alanine aminotransferase $(A L T)$, aspartate aminotransferase (AST), gamma-glutamyltransferase (GGT), hemoglobin and hematocrit, and white blood cell (WBC), red blood cell (RBC) and platelet (PLT) counts up to 12 months after the start of ARB or CCB monotherapy.

Results: We found a significant reduction of serum $\mathrm{TC}, \mathrm{HbA1C}$, hemoglobin and hematocrit and RBC count and a significant increase of serum potassium in ARB users, and a reduction of serum TC and hemoglobin in CCB users, from the baseline period to the exposure period. The reductions of RBC count, hemoglobin and hematocrit in ARB users were significantly greater than those in CCB users. The increase of serum potassium in ARB users was significantly greater than that in CCB users.

Conclusions: Our study suggested that hematological adverse effects and electrolyte imbalance are greater with ARB monotherapy than with CCB monotherapy.

Keywords: Angiotensin II receptor blocker (ARB), Calcium channel blocker (CCB), Hematological parameter, Retrospective observational study

\footnotetext{
* Correspondence: takahashi.yasuo@nihon-u.ac.jp

2Division of Clinical Trial Management, Advanced Medical Research Center, Nihon University School of Medicine, 30-1 Oyaguchi-Kamimachi, Itabashi-ku, Tokyo173-8610, Japan

Full list of author information is available at the end of the article
} 


\section{Introduction}

Angiotensin II type I receptor blockers (ARBs) are well established antihypertensive drugs that are frequently used as the first-line drug for hypertension. Recently, there has been a focus on the beneficial effects of ARBs other than their antihypertensive effect, such as reduction of proteinuria [1] and decreased heart failure risk in patients with chronic heart failure [2]. Calcium channel blockers (CCBs) are also widely used first-line antihypertensive drugs. CCBs are known to decrease the risk of coronary heart disease and non-fatal stroke in patients with hypertension [3], and to decrease proteinuria in patients with chronic renal disease [4]. While ARBs and CCBs have a favorable effect on blood pressure and decrease the risk of several complications, these drugs have some adverse effects. Renin-angiotensin system inhibitors including ARBs are known to cause hyperkalemia [5] and anemia [6,7]. CCBs are known to cause edema [8].

Hypertension and type 2 diabetes mellitus are conditions that frequently coexist [9], both of which carry an increased risk of cardiovascular and renal disease. Hypertension significantly hastens the progression of diabetic nephropathy and increases the risk of cardiovascular events or death in patients with diabetes. On the contrary, lowering blood pressure decreases albuminuria in type 2 diabetes $[10,11]$. On the other hand, ARBs have a beneficial effect that prevents the new-onset of diabetes [12], and there has been a recent focus on the effect of ARBs on glucose metabolism. We demonstrated a favorable effect of ARB monotherapy on glucose metabolism in non-diabetic hypertensive patients [13]. Whether ARBs have a favorable effect on laboratory parameters, including parameters of glucose metabolism in diabetic hypertensive patients, may be of clinical significance.

Some randomized clinical studies have compared the adverse effects of ARBs and CCBs. [14-16]. However, those studies usually focused on the adverse events of antihypertensive drugs, and there are few large-scale studies focused on the effects of the drugs on laboratory parameters. In addition, few studies have targeted ARB and $\mathrm{CCB}$ monotherapy using a clinical database reflecting 'real-world' data. Therefore, in this study, we evaluated and compared the effects of $\mathrm{ARB}$ and $\mathrm{CCB}$ monotherapy on laboratory parameters, including parameters of lipid metabolism, glucose metabolism, renal function, hepatic function and hematological analysis in patients with concomitant hypertension and type 2 diabetes mellitus, using a clinical database.

\section{Materials and methods Data source}

This was a retrospective database study using the Nihon University School of Medicine (NUSM) Clinical
Data Warehouse (CDW). NUSM's CDW is a centralized data repository that integrates separate databases, including an order entry database and a laboratory results database, from the hospital information systems at three hospitals affiliated to NUSM. The prescribing data of over 0.5 million patients are linked longitudinally to detailed clinical information such as patient demographics, diagnosis, and laboratory results data. The schema of NUSM's CDW has been reported by Takahashi et al. [17].

\section{Study population}

For this study, we identified type 2 diabetes mellitus patients with mild to moderate hypertension aged over 20 years, who had been newly treated with ARB monotherapy $(n=922)$ or dihydropyridine CCB monotherapy $(\mathrm{n}=731)$ for at least two months between Nov 1, 2004 and July 31, 2011. The antihypertensive drugs used in the $A R B$ and $C C B$ monotherapy groups are listed in Table 1. We compared new users of ARBs $(n=601)$ with propensity-score matched samples of new CCB users $(n=601)$. We excluded patients who had been treated with other antihypertensive drugs (ARB combination drug, angiotensin-converting enzyme inhibitor (ACEI), diuretic, alpha-blocker, beta-blocker, alpha and betablocker, alpha-agonist, reserpine, vasodilator, or renin inhibitor) during the study period. The experimental protocol was approved by the Ethical Committee of Nihon University School of Medicine.

\section{Exposure and measurements}

The baseline measurement period (non-exposure period) was defined as within 12 months before the start of ARB or CCB monotherapy. The exposure period (outcome measurement period) was defined as between 2 and 12 months after the start of ARB or CCB monotherapy. The mean exposure of ARB users and CCB users was 243.2 days and 242.1 days, respectively. Laboratory data, including serum levels of triglyceride (TG), total cholesterol (TC), non-fasting blood glucose, hemoglobin A1c (HbA1c), creatinine, sodium, potassium, alanine aminotransferase (ALT), aspartate aminotransferase (AST), and gamma-glutamyltransferase (GGT), hemoglobin, hematocrit, and white blood cell (WBC), red blood cell (RBC) and platelet (PLT) counts, were collected for each individual at the date nearest the start of ARB or CCB monotherapy in the baseline period, and at the date nearest 12 months after the start of ARB or CCB monotherapy in the exposure period.

\section{Data elements}

For each patient, we collected information of patient demographics (age and sex), medical history, use of medication, and laboratory results. Medical history included 
Table 1 Antihypertensive drugs

\begin{tabular}{|c|c|c|c|c|}
\hline \multirow[t]{2}{*}{ Category } & \multirow[t]{2}{*}{ Generic name } & \multirow[t]{2}{*}{ Trade name } & \multicolumn{2}{|c|}{ No. of cases of monothrapy } \\
\hline & & & Before PSmatching & After PSmatching \\
\hline \multicolumn{5}{|l|}{ ARBs } \\
\hline & candesartan cilexetil & Blopress & 289 & 200 \\
\hline & losartan potassium & Nu-lotan & 154 & 87 \\
\hline & olmesartan medoxomil & Olmetec & 177 & 113 \\
\hline & telmisartan & Micardis & 141 & 91 \\
\hline & valsartan & Diovan & 161 & 110 \\
\hline \multicolumn{5}{|l|}{ CCBs } \\
\hline & amlodipine besilate & Norvasc, Amlodin & 355 & 277 \\
\hline & azelnidipine & Calblock & 46 & 38 \\
\hline & benidipine hydrochloride & Coniel & 82 & 66 \\
\hline & cilnidipine & Atelec, Cinalong & 41 & 36 \\
\hline & manidipine hydrochloride & Calslot & 25 & 21 \\
\hline & nicardipine hydrochloride & Perdipine & 17 & 13 \\
\hline & nifedipine & Adalat, Herlat, Sepamit & 133 & 110 \\
\hline & nilvadipine & Nivadil & 30 & 24 \\
\hline & $\begin{array}{l}\text { others (barnidipine hydrochloride, efonidipine } \\
\text { hydrochloride ethanolate, felodipine, nitrendipine } \\
\text { and nisoldipine) }\end{array}$ & $\begin{array}{l}\text { Hypoca, Landel, Munobal, } \\
\text { Baylotensin, Baymycard }\end{array}$ & 22 & 16 \\
\hline
\end{tabular}

PS: propensity score.

cerebrovascular disease (ICD-10 code, I60-I69), ischemic heart disease (I20-I25), other heart disease (I30-I52), liver disease (K70-K77), kidney disease (N00-N19), gout (M10), thyroid gland disorder (E00-E07), rheumatoid arthritis (M05-M06), hyperlipidemia (E78.0-E78.5), or proteinuria diagnosed in the 365 days preceding the first date of prescription of $\mathrm{ARB}$ or $\mathrm{CCB}$. Drugs used during the 60 days before the start of $\mathrm{ARB}$ or $\mathrm{CCB}$ monotherapy included hypoglycemic drugs (including insulin and oral hypoglycemic drugs), lipid-lowering drugs (including statins, fibrates and other lipid-lowering drugs), diuretics, immunosuppressive drugs, gout drugs, potassium preparations, antipsychotics, chemotherapeutic drugs, steroids, non-steroidal anti-inflammatory drugs (NSAIDs), proton pump inhibitors, histamine $\mathrm{H}_{2}$ receptor blockers and thyroid drugs.

\section{Statistical analysis}

The ARB user group and CCB user group were matched by propensity score using a 5-digit, greedy 1:1 matching algorithm [18-20]. This method is the standard method to reduce bias by balancing covariates between settings, and has been used in many reports. To generate the propensity score, we used covariates including age, sex, medical history (cerebrovascular disease, ischemic heart disease, other heart disease, liver disease, kidney disease, gout, thyroid gland disorder, rheumatoid arthritis, hyperlipidemia and proteinuria) and previous drugs (hypoglycemic drugs including insulin and oral hypoglycemic drugs, lipid-lowering drugs including statins, fibrates and other lipid-lowering drugs, diuretics, immunosuppressive drugs, gout drugs, potassium preparations, antipsychotics, chemotherapeutic drugs, steroids, NSAIDs, proton pump inhibitors, histamine $\mathrm{H}_{2}$ receptor blockers and thyroid drugs), as listed in Table 2. We compared the prevalence of all baseline covariates before and after propensity score matching using $t$-test for continuous variables and chi-squared test for categorical data. After propensity score matching, covariance-adjusted and unadjusted generalized linear models (Dunnett-Hsu post-hoc analysis) were fitted to compare the mean values of laboratory parameters at baseline and during the exposure period in ARB users and CCB users, and were used to compare the mean change from the baseline value to the exposure value in ARB users and $\mathrm{CCB}$ users. The covariates that were used in the adjusted model included age, sex, medical history and previous medication, as listed in Table 2 . All reported $\mathrm{P}$ values of less than 0.05 were considered to indicate statistical significance. All statistical analyses were performed with SAS software, version 9.1.3 (SAS Institute Inc., Cary, NC).

\section{Results}

Table 2 shows the characteristics of the patients who had been treated with ARB monotherapy or CCB monotherapy, before and after propensity score matching. Before propensity score matching, ARB users were more likely to have thyroid disease, kidney disease, proteinuria and 
Table 2 Baseline characteristics before and after propensity score matching

\begin{tabular}{|c|c|c|c|c|c|c|}
\hline \multirow[t]{2}{*}{ Characteristics } & \multicolumn{3}{|c|}{ Before matching } & \multicolumn{3}{|c|}{ After macthing } \\
\hline & $\begin{array}{c}\text { ARB users } \\
(n=922)\end{array}$ & $\begin{array}{c}\text { CCB users } \\
(\mathrm{n}=731)\end{array}$ & $p$ value & $\begin{array}{c}\text { ARB users } \\
(n=601)\end{array}$ & $\begin{array}{c}\text { CCB users } \\
p \text { value }\end{array}$ & $(n=601)$ \\
\hline Age (mean, SE) & $61.7 \pm 0.4$ & $66.8 \pm 0.35$ & $<.0001 *$ & $65.5 \pm 0.4$ & $65.6 \pm 0.39$ & 0.8268 \\
\hline Age over 75 years & $130(14.1 \%)$ & $158(21.6 \%)$ & $<.0001 *$ & $110(18.3 \%)$ & $113(18.8 \%)$ & 0.8238 \\
\hline Women & $316(34.3 \%)$ & $281(38.4 \%)$ & 0.0798 & $224(37.3 \%)$ & $225(37.4 \%)$ & 0.9525 \\
\hline \multicolumn{7}{|l|}{ Medical history } \\
\hline Cerebrovascular disease & $254(27.5 \%)$ & $208(28.5 \%)$ & 0.6837 & $170(28.3 \%)$ & $185(30.8 \%)$ & 0.3429 \\
\hline Ischemic heart disease & $317(34.4 \%)$ & $297(40.6 \%)$ & $0.009 *$ & $228(37.9 \%)$ & $233(38.8 \%)$ & 0.7668 \\
\hline Other heart disease & $208(22.6 \%)$ & $193(26.4 \%)$ & 0.0703 & $156(26.0 \%)$ & $149(34.8 \%)$ & 0.6427 \\
\hline Gout & $28(3.0 \%)$ & $43(5.9 \%)$ & $0.0046 *$ & $25(4.2 \%)$ & $25(4.2 \%)$ & 1 \\
\hline Thyroid disorder & $266(28.9 \%)$ & $155(21.2 \%)$ & $0.0004^{*}$ & $141(23.5 \%)$ & $142(23.6 \%)$ & 0.9458 \\
\hline Rheumatoid arthritis & $85(9.2 \%)$ & $68(9.3 \%)$ & 0.9538 & $48(8.0 \%)$ & $51(8.5 \%)$ & 0.753 \\
\hline Liver disease & $481(52.2 \%)$ & $404(55.3 \%)$ & 0.2098 & $327(54.4 \%)$ & $316(52.6 \%)$ & 0.5247 \\
\hline Kidney disease & $688(74.6 \%)$ & $483(66.1 \%)$ & $0.0001 *$ & $407(67.7 \%)$ & $411(68.4 \%)$ & 0.8046 \\
\hline Hyperlipidemia & $860(93.3 \%)$ & $678(92.7 \%)$ & 0.6764 & $559(93.0 \%)$ & $561(93.3 \%)$ & 0.819 \\
\hline Proteinuria & $463(50.2 \%)$ & $298(40.8 \%)$ & $0.0001 *$ & $255(42.4 \%)$ & $256(42.6 \%)$ & 0.9535 \\
\hline \multicolumn{7}{|l|}{ Previous drugs } \\
\hline Hypoglycemic drugs & $226(24.5 \%)$ & $188(25.7 \%)$ & 0.574 & $148(24.6 \%)$ & $156(26.0 \%)$ & 0.5955 \\
\hline Insulin & $79(8.6 \%)$ & $44(6.0 \%)$ & $0.0498 *$ & $36(6.0 \%)$ & $42(7.0 \%)$ & 0.4823 \\
\hline Oral hypoglycemic drugs & $171(18.5 \%)$ & $160(21.9 \%)$ & 0.0918 & $126(21.0 \%)$ & $129(21.5 \%)$ & 0.8324 \\
\hline Lipid-lowering drugs & $339(36.8 \%)$ & $237(32.4 \%)$ & 0.0655 & $201(33.4 \%)$ & $200(33.3 \%)$ & 0.9512 \\
\hline Statin & $286(31.0 \%)$ & $200(27.4 \%)$ & 0.1048 & $171(28.5 \%)$ & $169(28.1 \%)$ & 0.8981 \\
\hline Fibrate & $36(3.9 \%)$ & $29(4.0 \%)$ & 0.9481 & $21(3.5 \%)$ & $23(3.8 \%)$ & 0.7587 \\
\hline Other lipid-lowering drugs & $30(3.3 \%)$ & $23(3.1 \%)$ & 0.902 & $18(3.0 \%)$ & $18(3.0 \%)$ & 1 \\
\hline Diuretics & $7(0.8 \%)$ & $1(0.1 \%)$ & 0.0701 & $1(0.2 \%)$ & $1(0.2 \%)$ & 1 \\
\hline Immunosuppressive drugs & $14(1.5 \%)$ & $10(1.4 \%)$ & 0.7995 & $5(0.8 \%)$ & $8(1.3 \%)$ & 0.4028 \\
\hline Gout drugs & $59(6.4 \%)$ & $86(11.8 \%)$ & $0.0001 *$ & $48(8.0 \%)$ & $51(8.5 \%)$ & 0.753 \\
\hline Potassium preparations & $2(0.2 \%)$ & $4(0.5 \%)$ & 0.2674 & $1(0.2 \%)$ & $0(0 \%)$ & 0.3171 \\
\hline Antipsychotics & $38(4.1 \%)$ & $19(2.6 \%)$ & 0.0921 & $18(3.0 \%)$ & $17(2.8 \%)$ & 0.8638 \\
\hline Chemotherapeutic drugs & $14(1.5 \%)$ & $18(2.5 \%)$ & 0.1666 & $11(1.8 \%)$ & $9(1.5 \%)$ & 0.652 \\
\hline Steroids & $57(6.2 \%)$ & $43(5.9 \%)$ & 0.7995 & $32(5.3 \%)$ & $33(5.5 \%)$ & 0.8985 \\
\hline NSAIDs & $284(30.8 \%)$ & $236(32.3 \%)$ & 0.5193 & $181(30.1 \%)$ & $190(31.6 \%)$ & 0.5741 \\
\hline Proton pump inhibitors & $126(13.7 \%)$ & $82(11.2 \%)$ & 0.136 & $77(12.8 \%)$ & $75(12.5 \%)$ & 0.8622 \\
\hline $\mathrm{H} 2$ blockers & $120(13.0 \%)$ & $144(19.7 \%)$ & $0.0002 *$ & $93(15.5 \%)$ & $100(16.6 \%)$ & 0.5824 \\
\hline Thyroid drugs & $12(1.3 \%)$ & $13(1.8 \%)$ & 0.4301 & $10(1.7 \%)$ & $9(1.5 \%)$ & 0.8171 \\
\hline
\end{tabular}

Data are numbers of individuals (\%) unless otherwise stated. Abbreviations: ARB; angiotensin II type I receptor blocker, CCB; calcium channel blocker, NSAID; nonsteroidal anti-inflammatory drug. *: $p<0.05$ (ARB vs CCB).

use insulin, and less likely to have ischemic heart disease, gout, use gout drugs and use $\mathrm{H} 2$ blockers than $\mathrm{CCB}$ users. After propensity score matching, the mean age was 65.5 and 65.6 years, and $37.3 \%$ and $37.4 \%$ of ARB users and CCB users were women, respectively.

Table 3 shows laboratory parameters at baseline and during the exposure period. In ARB users, the mean values of $\mathrm{TC}, \mathrm{HbA1c}$, hematocrit and hemoglobin and $\mathrm{RBC}$ count significantly decreased during the exposure period compared with those during the baseline period, after adjustment for age, sex, medical history and previous medication. The adjusted mean value of potassium significantly increased during the exposure period compared with that in the baseline period in ARB users. The adjusted mean values of TG, glucose, creatinine, sodium, ALT, AST, GGT and WBC and PLT counts were not significantly different during the exposure period compared with those in the baseline period in ARB users. In CCB 
Table 3 Unadjusted and adjusted mean $(95 \% \mathrm{CI})$ laboratory test values according to ARB or CCB use after propensity score matching

\begin{tabular}{|c|c|c|c|c|c|c|c|c|c|c|c|c|}
\hline \multirow{2}{*}{$\begin{array}{l}\text { Laboratory } \\
\text { test }\end{array}$} & \multicolumn{6}{|c|}{ ARBs $(n=601)$} & \multicolumn{6}{|c|}{ CCBs $(n=601)$} \\
\hline & Mean & $\begin{array}{l}\text { Unadjusted } \\
(95 \% \mathrm{Cl})\end{array}$ & p-value & Mean & $\begin{array}{l}\text { Adjusted } \dagger \\
(95 \% \mathrm{Cl})\end{array}$ & p-value & Mean & $\begin{array}{l}\text { Unadjusted } \\
(95 \% \mathrm{Cl})\end{array}$ & p-value & Mean & $\begin{array}{l}\text { Adjusted } \dagger \\
(95 \% \mathrm{Cl})\end{array}$ & $\mathrm{p}$-value \\
\hline \multicolumn{13}{|l|}{ TG (mmol/L) } \\
\hline baseline & 1.65 & $(1.56,1.73)$ & 0.5113 & 1.65 & $(1.56,1.73)$ & 0.4846 & 1.63 & $(1.56,1.71)$ & 0.2961 & 1.63 & $(1.56,1.7)$ & 0.2604 \\
\hline exposure & 1.60 & $(1.52,1.69)$ & & 1.6 & $(1.52,1.69)$ & & 1.57 & $(1.5,1.65)$ & & 1.57 & $(1.5,1.65)$ & \\
\hline \multicolumn{13}{|l|}{$\mathrm{TC}(\mathrm{mmol} / \mathrm{L})$} \\
\hline baseline & 5.20 & $(5.12,5.28)$ & $0.0056 *$ & 5.2 & $(5.13,5.27)$ & $0.0018 *$ & 5.18 & $(5.1,5.26)$ & $0.0351 *$ & 5.18 & $(5.11,5.25)$ & $0.0206 *$ \\
\hline exposure & 5.05 & $(4.97,5.12)$ & & 5.05 & $(4.98,5.12)$ & & 5.06 & $(4.98,5.14)$ & & 5.06 & $(4.99,5.13)$ & \\
\hline \multicolumn{13}{|c|}{ Blood glucose (mmol/L) } \\
\hline baseline & 7.88 & $(7.63,8.13)$ & 0.3744 & 7.88 & $(7.66,8.1)$ & 0.3133 & 7.96 & $(7.7,8.23)$ & 0.3809 & 7.96 & $(7.72,8.2)$ & 0.3257 \\
\hline exposure & 7.72 & $(7.47,7.97)$ & & 7.72 & $(7.5,7.94)$ & & 7.79 & $(7.53,8.06)$ & & 7.79 & $(7.55,8.03)$ & \\
\hline \multicolumn{13}{|l|}{$\mathrm{HbA1c}(\%)$} \\
\hline baseline & 6.97 & $(6.86,7.08)$ & $0.0451 *$ & 6.97 & $(6.88,7.05)$ & $0.0074 *$ & 6.93 & $(6.81,7.05)$ & 0.2981 & 6.93 & $(6.84,7.02)$ & 0.1793 \\
\hline exposure & 6.81 & $(6.7,6.92)$ & & 6.81 & $(6.73,6.89)$ & & 6.84 & $(6.73,6.96)$ & & 6.84 & $(6.75,6.93)$ & \\
\hline \multicolumn{13}{|c|}{ Creatinine $(\mu \mathrm{mol} / \mathrm{L})$} \\
\hline baseline & 72.8 & $(70,75.5)$ & 0.2045 & 72.8 & $(70.7,74.8)$ & 0.0934 & 73.8 & $(70,77.7)$ & 0.5707 & 73.8 & $(71.3,76.4)$ & 0.3887 \\
\hline exposure & 75.3 & $(72.5,78)$ & & 75.3 & $(73.2,77.3)$ & & 75.4 & $(71.5,79.2)$ & & 75.4 & $(72.9,77.9)$ & \\
\hline \multicolumn{13}{|c|}{ Sodium (mmol/L) } \\
\hline baseline & 141.4 & $(141.2,141.6)$ & 0.1841 & 141.4 & $(141.2,141.6)$ & 0.1725 & 141.9 & $(141.7,142.1)^{\prime}$ & 0.1137 & 141.9 & $(141.7,142.1)$ & 0.0993 \\
\hline exposure & 141.2 & $(141,141.4)$ & & 141.2 & $(141,141.4)$ & & 141.7 & $(141.5,141.9)$ & & 141.7 & $(141.5,141.9)$ & \\
\hline \multicolumn{13}{|c|}{ Potassium (mmol/L) } \\
\hline baseline & 4.39 & $(4.36,4.42)$ & $0.0351 *$ & 4.39 & $(4.36,4.42)$ & $0.0241 *$ & 4.3 & $(4.26,4.33)$ & 0.8344 & 4.3 & $(4.26,4.33)$ & 0.8298 \\
\hline exposure & 4.44 & $(4.41,4.47)$ & & 4.44 & $(4.41,4.47)$ & & 4.29 & $(4.26,4.32)$ & & 4.29 & $(4.26,4.32)$ & \\
\hline \multicolumn{13}{|l|}{$\mathrm{ALT}(\mathrm{U} / \mathrm{L})$} \\
\hline baseline & 27.2 & $(25.5,28.8)$ & 0.0903 & 27.2 & $(25.7,28.6)$ & 0.0577 & 28.5 & $(26.4,30.7)$ & 0.6781 & 28.5 & $(26.6,30.5)$ & 0.6465 \\
\hline exposure & 25.2 & $(23.5,26.8)$ & & 25.2 & $(23.7,26.6)$ & & 27.9 & $(25.8,30)$ & & 27.9 & $(26,29.8)$ & \\
\hline \multicolumn{13}{|l|}{ AST (U/L) } \\
\hline baseline & 27.3 & $(26,28.6)$ & 0.3521 & 27.3 & $(26.1,28.4)$ & 0.3041 & 28.2 & $(26.4,30)$ & 0.6099 & 28.2 & $(26.6,29.9)$ & 0.5741 \\
\hline exposure & 26.4 & $(25.1,27.7)$ & & 26.4 & $(25.2,27.6)$ & & 28.9 & $(27.1,30.7)$ & & 28.9 & $(27.2,30.5)$ & \\
\hline \multicolumn{13}{|l|}{ GGT (U/L) } \\
\hline baseline & 53.2 & $(46.8,59.6)$ & 0.4179 & 53.2 & $(47.1,59.4)$ & 0.3967 & 56.1 & $(49.3,62.8)$ & 0.7434 & 56.1 & $(49.7,62.5)$ & 0.7292 \\
\hline exposure & 49.5 & $(43.1,55.9)$ & & 49.5 & $(43.4,55.6)$ & & 57.7 & $(50.9,64.4)$ & & 57.7 & $(51.3,64.1)$ & \\
\hline
\end{tabular}


Table 3 Unadjusted and adjusted mean $(95 \% \mathrm{CI})$ laboratory test values according to ARB or CCB use after propensity score matching (Continued)

\begin{tabular}{|c|c|c|c|c|c|c|c|c|c|c|c|c|}
\hline \multicolumn{13}{|l|}{$\overline{W B C}\left(\times 10^{9} / L\right)$} \\
\hline baseline & 6.36 & $(6.21,6.51)$ & 0.8579 & 6.36 & $(6.22,6.5)$ & 0.8494 & 6.47 & $(6.32,6.63)$ & 0.6118 & 6.47 & $(6.33,6.62)$ & 0.5847 \\
\hline exposure & 6.34 & $(6.19,6.49)$ & & 6.34 & $(6.2,6.48)$ & & 6.42 & $(6.26,6.57)$ & & 6.42 & $(6.27,6.56)$ & \\
\hline \multicolumn{13}{|l|}{$\mathrm{RBC}\left(\times 10^{12} / \mathrm{L}\right)$} \\
\hline baseline & 4.36 & $(4.32,4.4)$ & $0.0015 *$ & 4.36 & $(4.33,4.4)$ & $0.0002 *$ & 4.4 & $(4.36,4.45)$ & 0.3324 & 4.4 & $(4.36,4.45)$ & 0.2798 \\
\hline exposure & 4.26 & $(4.22,4.31)$ & & 4.26 & $(4.23,4.3)$ & & 4.37 & $(4.33,4.42)$ & & 4.37 & $(4.33,4.41)$ & \\
\hline \multicolumn{13}{|l|}{$\operatorname{PLT}\left(\times 10^{9} / \mathrm{L}\right)$} \\
\hline baseline & 221.2 & $(216.4,226)$ & 0.8006 & 221.2 & $(216.8,225.6)$ & 0.7845 & 222.4 & $(216.8,227.9)$ & 0.2089 & 222.4 & $(217.2,227.6)$ & 0.1825 \\
\hline exposure & 222.1 & $(217.3,226.9)$ & & 222.1 & $(217.7,226.5)$ & & 227.4 & $(221.9,233)$ & & 227.4 & $(222.2,232.6)$ & \\
\hline \multicolumn{13}{|c|}{ Hemoglobin (g/L) } \\
\hline baseline & 138.0 & $(136.7,139.4)$ & $0.0024 *$ & 138 & $(136.9,139.1)$ & $0.0002 *$ & 138.5 & $(137.1,139.8)$ & 0.0727 & 138.5 & $(137.4,139.6)$ & 0.0315 * \\
\hline exposure & 135.1 & $(133.8,136.5)$ & & 135.1 & $(134,136.2)$ & & 136.7 & $(135.4,138.1)$ & & 136.7 & $(135.6,137.9)$ & \\
\hline \multicolumn{13}{|c|}{ Hematocrit $(\mathrm{mmol} / \mathrm{mol})$} \\
\hline baseline & 0.407 & $(0.404,0.411)$ & $0.0069 *$ & 0.407 & $(0.404,0.411)$ & $0.0012 *$ & 0.409 & $(0.405,0.413)$ & 0.285 & 0.409 & $(0.406,0.412)$ & 0.2033 \\
\hline exposure & 0.400 & $(0.396,0.404)$ & & 0.4 & $(0.397,0.403)$ & & 0.406 & $(0.402,0.41)$ & & 0.406 & $(0.403,0.409)$ & \\
\hline
\end{tabular}

count, PLT; platelet count. *: $\mathrm{p}<0.05$ (baseline vs exposure). † Analyses were adjusted by covariates including age, sex, medical history and previous medication 
Table 4 Unadjusted and adjusted mean changes in laboratory parameters values during0020exposure period from baseline Laboratory

test

$\Delta \mathrm{TG}(\mathrm{mmol} / \mathrm{L})$

$\mathrm{CCB}$

ARB

$-0.041$

$\begin{array}{ll}\text { CCB } & -0.119 \\ \text { ARB } & -0.153\end{array}$

$\triangle$ Blood glucose $(\mathrm{mmol} / \mathrm{L})$

$\begin{array}{cc}\text { CCB } & -0.17 \\ \text { ARB } & -0.161\end{array}$

$\triangle \mathrm{HbA1c}(\%)$

$C C B$

ARB

$-0.087$

$\Delta$ Creatinine $(\mu \mathrm{mol} / \mathrm{L})$

$\begin{array}{ll}\text { CCB } & 1.575 \\ \text { ARB } & 2.508\end{array}$

$\Delta$ Sodium $(\mathrm{mmol} / \mathrm{L})$

$\begin{array}{ll}\text { CCB } & -0.24 \\ \text { ARB } & -0.203\end{array}$

$\triangle$ Potassium $(\mathrm{mmol} / \mathrm{L})$

$\begin{array}{cc}\text { CCB } & -0.005 \\ \text { ARB } & 0.05\end{array}$

$\triangle \mathrm{ALT}(\mathrm{U} / \mathrm{L})$

CCB

ARB

$-0.639$

$\triangle \mathrm{AST}(\mathrm{U} / \mathrm{L})$

CCB

0.667

$-0.867$

$\Delta \mathrm{GGT}(\mathrm{U} / \mathrm{L})$

$$
\text { CCB }
$$

ARB

1.599

$-3.74$

$(-4.675,7.873)$

$\triangle \mathrm{WBC}\left(\times 10^{9} / \mathrm{L}\right)$

$\begin{array}{ll}\text { CCB } & -0.057 \\ \text { ARB } & -0.019\end{array}$

$\triangle \mathrm{RBC}\left(\mathrm{x} 10^{12} / \mathrm{L}\right)$

CCB

$-0.032$

$\triangle \mathrm{PLT}\left(\mathrm{X} 10^{9} / \mathrm{L}\right)$

$C C B$

$A R B$

$$
5.03
$$

0.872

$\Delta$ Hemoglobin $(\mathrm{g} / \mathrm{L})$

$\begin{array}{ll}\text { CCB } & -1.722 \\ \text { ARB } & -2.894\end{array}$

$(-0.116,0.035)$

$(-0.182,-0.056)$

$(-0.217,-0.09)$

$(-0.439,0.099)$

$(-0.431,0.108)$

$(-0.175,0.002)$

$(-0.246,-0.069)$

$(-0.59,3.74)$

$(0.343,4.673)$

$(-0.462,-0.017)$

$(-0.425,0.019)$

$(-0.037,0.027)$

$(0.018,0.082)$

$(-2.463,1.185)$

$(-3.835,-0.188)$

$(-0.859,2.193)$

$(-2.393,0.659)$

$(-10.015,2.534)$

$(-0.185,0.072)$

$(-0.148,0.109)$

$(-0.058,-0.006)$

$(-0.123,-0.072)$

p-value

0.7509

0.4512

$-0.149$

0.9651

$-0.177$

$-0.154$

0.2669

$-0.089$

$-0.155$

0.5503

1.667

2.416

0.8194

$-0.239$

$-0.204$

0.0173 *

$-0.005$

0.05

0.2966

$-0.633$

$-2.018$

0.1633

0.664

$-0.864$

0.238

1.648

$-3.789$

0.6864

$-0.048$

$-0.028$

0.0005 *

$-0.032$

$-0.098$

0.0825

$(-2.448,4.192)$

$(-2.542,-0.903)$

$(-3.713,-2.074)$

0.845

0.0476 *

$-1.721$

$-2.895$
Adjusted $\dagger$

(95\%Cl)

p-value

$(-0.139,0.006)$

$(-0.105,0.04)$

0.5062

$(-0.185,-0.061)$

0.5664

$(-0.211,-0.087)$

$(-0.447,0.093)$

0.9085

$(-0.424,0.115)$

$(-0.175,-0.003)$

0.2887

$(-0.241,-0.069)$

$(-0.466,3.801)$

0.6275

$(0.282,4.549)$

$(-0.458,-0.019)$

0.8275

$(-0.424,0.016)$

$(-0.037,0.027)$

$0.0182 *$

$(0.018,0.081)$

$(-2.433,1.167)$

0.2871

$(-3.818,-0.218)$

$(-0.847,2.175)$

0.1618

$(-2.375,0.647)$

$(-4.644,7.939)$

0.2319

$(-10.081,2.502)$

$(-0.176,0.08)$

0.8298

$(-0.156,0.099)$

$(-0.058,-0.006)$

0.0004 *

$(-0.124,-0.072)$

$(1.793,8.321)$

0.0743

$(-2.419,4.109)$

$(-2.538,-0.904)$

0.047 * 
Table 4 Unadjusted and adjusted mean changes in laboratory parameters values during0020exposure period from baseline (Continued)

\begin{tabular}{|c|c|c|c|c|c|c|}
\hline \multicolumn{7}{|c|}{$\Delta$ Hematocrit $(\mathrm{mmol} / \mathrm{mol})$} \\
\hline$C C B$ & -0.003 & $(-0.005,-0.001)$ & $0.0103 *$ & -0.003 & $(-0.005,-0.001)$ & $0.0092 *$ \\
\hline$A R B$ & -0.007 & $(-0.01,-0.005)$ & & -0.007 & $(-0.01,-0.005)$ & \\
\hline
\end{tabular}

$\triangle$ indicates mean change in laboratory test value between baseline and exposure period. Abbreviations: TG; triglyceride, TC; total cholesterol, HbA1c; hemoglobin A1c, ALT; alanine aminotransferase, AST; asparate aminotransferase, GGT; $\gamma$-glutamyltransferase, WBC; white blood cell count, RBC; red blood cell count, PLT; platelet count. *: $p<0.05$ (ARB vs CCB). $†$ Analyses were adjusted by covariates including age, sex, medical history and previous drugs.

users, the adjusted mean values of TC and hemoglobin significantly decreased during the exposure period compared with those in the baseline period. The adjusted mean values of TG, glucose, HbA1c, sodium, creatinine, potassium, ALT, AST, GGT, hematocrit, and WBC, RBC and PLT counts were not significantly different during the exposure period compared with those in the baseline period in CCB users.

Table 4 shows the mean changes in laboratory parameters during the exposure period compared with the baseline period. The change in potassium was significantly greater in ARB users compared with $\mathrm{CCB}$ users, and the changes in RBC count, hemoglobin and hematocrit were significantly smaller in ARB users compared with CCB users after adjustment for covariates.

We further analyzed the data divided by sex, because the standard values of hemoglobin, hematocrit and RBC count differ by sex. Table 5 shows the mean changes in laboratory parameters during the exposure period compared with the baseline period after adjustment for covariates, in subclass analysis. In women, the change in potassium was significantly greater in ARB users than in $\mathrm{CCB}$ users, and the changes in hemoglobin, hematocrit and RBC count were significantly smaller in $A R B$ users than in CCB users. In men, the mean change in $R B C$ count was significant smaller in ARB users than in CCB users.

\section{Discussion}

In this study, we evaluated and compared the effects of $A R B$ and $C C B$ monotherapy on biochemical parameters including serum TG, TC, non-fasting blood glucose, HbA1c, sodium, potassium, creatinine, ALT, AST and GGT and hematological parameters including hemoglobin, hematocrit, and WBC, RBC and PLT counts in patients with mild to moderate hypertension and type 2 diabetes mellitus. We found a significant reduction of serum TC, HbA1c, hemoglobin, hematocrit and RBC count in ARB users, and a reduction of serum TC and hemoglobin level in CCB users, from the baseline period to during the exposure period. The reductions of $\mathrm{RBC}$ count, hemoglobin and hematocrit in ARB users were significantly greater than those in $\mathrm{CCB}$ users. The increase of serum potassium level in $A R B$ users was significantly greater than that in CCB users. These results suggest that hematological adverse effects and electrolyte imbalance are greater with ARB monotherapy than with CCB monotherapy.

It is known that renin-angiotensin system inhibitors, ACEIs and ARBs, occasionally cause anemia, while having protective effects on various organs. Valsartan decreases hematocrit in recipients of kidney transplantation [21]. Losartan decreases hematocrit, hemoglobin and erythrocyte count in recipients of kidney transplantation [6,22]. In animals, candesartan decreases hematocrit, hemoglobin, erythrocyte count, and erythropoietin level in the rat [23].Confirming these previous reports, our 'real-world' study showed adverse effects of ARB monotherapy on hemoglobin, hematocrit and RBC count.

There are some reports that the use of renin-angiotensin system inhibitors, including ARBs, is associated with hyperkalemia. The serum level of potassium is significantly higher in ARB users than in CCB users after renal transplantation [24]. The relative risk of hyperkalemia was 2-fold higher with dual therapy (ARB plus ACEI) than with monotherapy (ARB or ACEI) [25]. Use of ARBs and ACEIs is associated with a high prevalence of hyperkalemia, and the prevalence of hyperkalemia is significantly higher in ARB users than in ACEI users [5]. Supporting these previous reports of hyperkalemia, our study showed that ARB monotherapy caused electrolyte imbalance with respect to the serum level of potassium. Our study, in combination with previous reports, suggested that regular checks of serum potassium level may be advisable in ARB users.

There are few reports of ARBs affecting hepatic function. In patients with hypertension and abdominal obesity, there was no significant difference in the levels of ALT, AST and GGT between the candesartan group and placebo [26]. There was no significant difference in the levels of ALT and AST from baseline to six months of use of losartan in hypertensive diabetic patients [27]. Supporting these reports, there was no statistically significant difference in the serum levels of ALT and AST between baseline and the exposure period in both ARB users and CCB users in our study. In addition, those changes from baseline to during the exposure period were not significantly different between $A R B$ and $C C B$ users. Therefore, the influence of $A R B$ and 
Table 5 Adjusted mean changes in laboratory parameters during exposure period from baseline by sex

Laboratory

test

$\Delta \mathrm{TG}(\mathrm{mmol} / \mathrm{L})$

$\begin{array}{ll}\text { CCB } & -0.076 \\ \text { ARB } & -0.007\end{array}$

$\Delta \mathrm{TC}(\mathrm{mmol} / \mathrm{L})$

$\begin{array}{lll}\text { CCB } & -0.127 & (-0.239,-0.014) \\ \text { ARB } & -0.214 & (-0.326,-0.101)\end{array}$

$\triangle$ Blood glucose (mmol/L)

$\begin{array}{ll}\text { CCB } & -0.17 \\ \text { ARB } & -0.293\end{array}$

$(-0.593,0.253)$

$(-0.717,0.131)$

$\triangle \mathrm{HbA} 1 \mathrm{c}(\%)$

$\begin{array}{ll}\text { CCB } & -0.075 \\ \text { ARB } & -0.257\end{array}$

$(-0.216,0.066)$

$(-0.398,-0.116)$

$\Delta$ Creatinine $(\mu \mathrm{mol} / \mathrm{L})$

$\begin{array}{ll}\text { CCB } & 0.475 \\ \text { ARB } & 2.202\end{array}$

$(-0.776,1.726)$

$(0.948,3.456)$

$\Delta$ Sodium (mmol/L)

$\begin{array}{ll}\text { CCB } & -0.09 \\ \text { ARB } & -0.49\end{array}$

$(-0.434,0.254)$

$(-0.835,-0.145)$

$\Delta$ Potassium (mmol/L)

$\begin{array}{ll}\text { CCB } & -0.015 \\ \text { ARB } & 0.075\end{array}$

$\triangle \mathrm{ALT}(\mathrm{U} / \mathrm{L})$

$\begin{array}{ll}\text { CCB } & -0.92 \\ \text { ARB } & -3.32\end{array}$

$\triangle \mathrm{AST}(\mathrm{U} / \mathrm{L})$

CCB
ARB

$$
1.125
$$$$
-2.43
$$

$(-0.067,0.038)$

$(0.023,0.128)$

$(-3.478,1.636)$

$(-5.883,-0.758)$

$(-1.37,3.621)$

$(-4.931,0.072)$

$\Delta \mathrm{GGT}(\mathrm{U} / \mathrm{L})$

$\begin{array}{ll}\text { CCB } & -1.498 \\ \text { ARB } & -4.794\end{array}$

$(-6.821,3.824)$

$(-10.129,0.541)$

$\triangle \mathrm{WBC}\left(\times 10^{9} / \mathrm{L}\right)$

$\begin{array}{ll}\text { CCB } & -0.092 \\ \text { ARB } & 0.023\end{array}$

$\triangle \mathrm{RBC}\left(\mathrm{x} 10^{12} / \mathrm{L}\right)$

$$
\text { CCB }
$$

ARB

$-0.025$

$-0.12$

$\Delta \mathrm{PLT}\left(\times 10^{9} / \mathrm{L}\right)$

$$
\text { CCB }
$$

ARB

$$
4.675
$$

$-0.008$

$\Delta$ Hemoglobin $(\mathrm{g} / \mathrm{L})$

$\begin{array}{ll}\text { CCB } & -1.286 \\ \text { ARB } & -3.333\end{array}$

$(-0.278,0.094)$

$(-0.164,0.209)$

$(-0.061,0.012)$

$(-0.157,-0.083)$

$(-5.083,5.067)$

$(-2.417,-0.156)$

$(-4.466,-2.2)$
$(-0.389,9.738)$

p-value

0.3449

0.2907

$-0.115$

0.6908

$-0.152$

$-0.101$

0.0777

$-0.092$

$-0.1$

0.0592

2.346

2.578

0.1124

$-0.279$

$-0.082$

0.0188 *

0.0002

0.035

$-0.31$

$-1.393$

0.0796

0.59

$-0.135$

0.3965

3.176

$-2.839$

0.3973

$-0.02$

$-0.06$

0.0004 *

$-0.032$

$-0.088$

0.2057

4.86

1.776

0.0135 *

$-1.811$

$-2.804$
Adjusted Men

(95\%Cl)

p-value

$(-0.162,0.039)$

$(-0.148,0.053)$

0.8484

$(-0.19,-0.044)$

0.9665

$(-0.188,-0.042)$

$(-0.505,0.202)$

0.8447

$(-0.454,0.252)$

$(-0.201,0.016)$

0.9247

$(-0.208,0.009)$

$(-0.967,5.658)$

0.923

$(-0.73,5.886)$

$(-0.567,0.009)$

0.3479

$(-0.37,0.206)$

$(-0.041,0.041)$

0.2423

$(-0.006,0.076)$

$(-2.767,2.147)$

0.5436

$(-3.847,1.06)$

$(-1.334,2.514)$

0.6034

$(-2.057,1.786)$

$(-6.48,12.832)$

0.3908

$(-12.482,6.804)$

$(-0.191,0.152)$

0.7449

$(-0.232,0.111)$

$(-0.067,0.003)$

0.0286 *

$(-0.123,-0.053)$

$(0.551,9.17)$

0.3242

$(-2.528,6.08)$

$(-2.931,-0.692)$

0.2222 
Table 5 Adjusted mean changes in laboratory parameters during exposure period from baseline by sex (Continued)

\begin{tabular}{|c|c|c|c|c|c|c|}
\hline \multicolumn{7}{|c|}{$\overline{\Delta \text { Hematocrit }(\mathrm{mmol} / \mathrm{mol})}$} \\
\hline$C C B$ & -0.002 & $(-0.006,0.001)$ & 0.0076 * & -0.003 & $(-0.006,0.0004)$ & 0.0796 \\
\hline ARB & -0.009 & $(-0.012,-0.005)$ & & -0.007 & $(-0.01,-0.004)$ & \\
\hline
\end{tabular}

$\triangle$ indicates mean change in laboratory test value between baseline and exposure period. Abbreviations: TG; triglyceride, TC; total cholesterol, HbA1c; hemoglobin A1c, ALT; alanine aminotransferase, AST; asparate aminotransferase, GGT; $\gamma$-glutamyltransferase, WBC; white blood cell count, RBC; red blood cell count, PLT; platelet count. * $\mathrm{p}<0.05$ (ARB vs $\mathrm{CCB}$ ). + Analyses were adjusted by covariates including age, sex, medical history and previous drugs.

CCB monotherapy on hepatic function may be minimal and not of clinical concern.

TC and HbA1c levels in ARB users decreased during the exposure period compared to the baseline period in this study. Some ARBs modulate peroxisome proliferator-activated receptor- $\gamma$ (PPAR- $\gamma$ ), which regulates lipid metabolism and is associated with insulin resistance $[28,29]$. There are some reports that telmisartan, which is a strong modulator of PPAR- $\gamma$, has a favorable effect on glucose metabolism. Telmisartan significantly improved HOMA-IR in hypertensive patients and also significantly decreased $\mathrm{HbA} 1 \mathrm{c}$ in type 2 diabetic patients, especially in those with poor glycemic control [30]. Treatment with telmisartan significantly improved the hyper-insulin response to glucose loading in patients with hypertension and obesity showing insulin resistance [31]. The favorable effect of ARBs on lipid and glucose metabolism that we observed may be caused in part by activation of PPAR- $\gamma$. Another reason for the decrease in HbA1c level in ARB users in our study may be the effect of the reduction of hemoglobin level. Sinha et al. suggested that both serum hemoglobin and HbA1c levels are significantly increased in patients with treatment of iron-deficiency anemia [32]. Ford et al. suggested that hemoglobin concentration is positively correlated with the concentration of HbA1c [33]. The effect of ARBs on the HbA1c level that we observed may have been partly influenced by the reduction of hemoglobin level.

There was no statistically significant difference in the level of blood glucose between the baseline and exposure periods in ARB users; however, we have previously reported that ARB monotherapy decreases the level of non-fasting blood glucose during a 6-month exposure period in non-diabetic patients with hypertension [13]. This discrepancy could be explained in part by differences in the duration of treatment or history of diabetes mellitus. It is possible that the glucose-lowering effect of ARB monotherapy could be weaker in patients with diabetes mellitus than in non-diabetic patients. We will evaluate these issues in our next study.

A decrease of TC was also observed in CCB users in our study. Nakamura et al. reported that CCBs decrease TC in patients with CKD [34]. Supporting the previous report, our results revealed a beneficial effect on lipid metabolism in CCB users in patients with hypertension and type 2 diabetes mellitus.
Subclass analysis showed that the reduction of $\mathrm{RBC}$ count was significantly greater in ARB users than in $\mathrm{CCB}$ users, in both men and women. On the other hand, the mean changes of potassium, hemoglobin and hematocrit in women were significantly different between ARB users and CCB users, but were not significantly different in men (Table 5). The reason for this discrepancy may be as follows. First, the effects of ARBs on hematological parameters are stronger in patients with low hemoglobin and hematocrit than in those with high levels. It is well known that there is a sex difference in hematological parameters; RBC count, hemoglobin and hematocrit are generally lower in women than in men. Second, the effect of ARBs on hemoglobin and hematocrit may reflect their effects on hormones. Testosterone is known to increase hemoglobin and hematocrit [35]. However, the reason for this discrepancy between women and men is still unclear.

Our study has several limitations. First, the retrospective and non-randomized nature of the design involved inherent issues of selection bias and confounding. We used rigorous statistical methods to balance potential confounding variables between ARB and CCB users, including propensity score matching. However, their ability to control for differences was limited to variables that were available or measurable. Second, we compared the effects of ARBs and CCBs in this study. However, the effects of ARBs on lipid and glucose metabolism slightly differ among these drugs [36-38], and further studies are needed to compare the effects of individual drugs. Third, we did not fix the daily dosage in both $A R B$ and $C C B$ users, because the achievement of blood pressure goal requires various doses of an agent across different individuals or even in the same individual in clinical practice. This study was not designed to assess the effects of ARBs and CCBs at each dosage, because it is difficult to determine whether or not pharmacodynamics are dosedependent in clinical settings. However, the findings of our study, using a sophisticated statistical method in a real-world setting, are reliable and informative for clinicians.

\section{Conclusions}

In this study, we observed greater reductions of hemoglobin, hematocrit and RBC count, and a greater increase of serum potassium level in patients who had 
received ARB monotherapy compared with $\mathrm{CCB}$ monotherapy. We observed significant differences between $A R B$ and $C C B$ users, although the mean values of these parameters remained within normal limits during the baseline and exposure periods. On the other hand, there was no significant difference in parameters of lipid metabolism, glucose metabolism and hepatic function and WBC and PLT counts between ARB and CCB users. Our findings support the clinical evidence that ARB therapy is associated with hematological adverse effects and electrolyte imbalance.

\section{Abbreviations}

ARB: Angiotensin II type I receptor blocker; CCB: Calcium channel blocker; NUSM: Nihon University School of Medicine; CDW: Clinical Data Warehouse; ACEl: Angiotensin-converting enzyme inhibitor; TG: Triglyceride; TC: Total cholesterol; HbA1c: Hemoglobin A1c; ALT: Alanine aminotransferase; AST: Aspartate aminotransferase; GGT: Gamma-glutamyltransferase; WBC: White blood cell; RBC: Red blood cell; PLT: Platelet; NSAID: Nonsteroidal anti-inflammatory drug; PPAR-ү: Peroxisome proliferator-activated receptor-ү; PS: Propensity score.

\section{Competing interests}

The authors declare that they have no competing interest.

\section{Acknowledgement}

This work was supported in part by Tempstaff Co., Ltd. (Tokyo, Japan).

\section{Author details}

'Division of Genomic Epidemiology and Clinical Trials, Advanced Medical Research Center, Nihon University School of Medicine, 30-1 OyaguchiKamimachi, Itabashi-ku, Tokyo173-8610, Japan. ${ }^{2}$ Division of Clinical Trial Management, Advanced Medical Research Center, Nihon University School of Medicine, 30-1 Oyaguchi-Kamimachi, Itabashi-ku, Tokyo173-8610, Japan. ${ }^{3}$ Division of Pharmacology, Department of Biomedical Sciences, Nihon University School of Medicine, 30-1 Oyaguchi-Kamimachi, Itabashi-ku, Tokyo173-8610, Japan. ${ }^{4}$ Division of Laboratory Medicine, Department of Pathology and Microbiology, Nihon University School of Medicine, 30-1 Oyaguchi-Kamimachi, Itabashi-ku, Tokyo173-8610, Japan.

\section{Authors' contributions}

YN and YT conceived the study and participated in its design. YN performed the statistical analyses. YN and YT drafted the manuscript. TN and SA interpreted the data. All authors have read and approved the final manuscript.

Received: 11 February 2012 Accepted: 07 April 2012

Published: 17 May 2012

\section{References}

1. Kunz R, Friedrich C, Wolbers M, Mann JF: Meta-analysis: effect of monotherapy and combination therapy with inhibitors of the renin angiotensin system on proteinuria in renal disease. Ann Intern Med 2008, 148:30-48.

2. Pfeffer MA, Swedberg K, Granger CB, Held P, McMurray JJ, Michelson EL, Olofsson B, Ostergren J, Yusuf S, Pocock S, CHARM Investigators and Committees: Effects of candesartan on mortality and morbidity in patients with chronic heart failure: the CHARM-Overall programme. Lancet 2003, 362:759-766.

3. Dahlöf B, Sever PS, Poulter NR, Wedel H, Beevers DG, Caulfield M, Collins R, Kjeldsen SE, Kristinsson A, McInnes GT, Mehlsen J, Nieminen M, O'Brien E, Ostergren J, ASCOT Investigators: Prevention of cardiovascular events with an antihypertensive regimen of amlodipine adding perindopril as required versus atenolol adding bendroflumethiazide as required, in the Anglo-Scandinavian Cardiac Outcomes Trial-Blood Pressure Lowering Arm (ASCOT-BPLA): a multicentre randomised controlled trial. Lancet 2005, 366:895-906
4. Fujita T, Ando K, Nishimura H, Ideura T, Yasuda G, Isshiki M, Takahashi K, Cilnidipine versus Amlodipine Randomised Trial for Evaluation in Renal Disease (CARTER) Study Investigators: Antiproteinuric effect of the calcium channel blocker cilnidipine added to renin-angiotensin inhibition in hypertensive patients with chronic renal disease. Kidney Int 2007, 72:1543-1549.

5. Sadjadi SA, MCMillan Jl, Jaipaul N, Blakely P, Hline SS: A comparative study of the prevalence of hyperkalemia with the use of angiotensin-converting enzyme inhibitors versus angiotensin receptor blockers. Ther Clin Risk Manag 2009, 5:547-552.

6. Ersoy A, Kahvecioglu S, Ersoy C, Cift A, Dilek K: Anemia due to losartan in hypertensive renal transplant recipients without posttransplant erythrocytosis. Transplant Proc 2005, 37:2148-2150.

7. López V, Martin M, Cobelo C, Aranda P, Cabello M, Sola E, Gutierrez C, Burgos D, Martínez D, Hernandez D: Renin-angiotensin system dual blockade using angiotensin receptor plus aliskiren decreases severe proteinuria in kidney transplant recipients. Transplant Proc 2010, 42:2883-2885.

8. Chrysant SG: Proactive compared with passive adverse event recognition: calcium channel blocker-associated edema. J Clin Hypertens (Greenwich) 2008, 10:716-722.

9. Mancia G, De Backer G, Dominiczak A, Cifkova R, Fagard R, Germano G, Grassi G, Heagerty AM, Kjeldsen SE, Laurent S: 2007 Guidelines for the Management of Arterial Hypertension: The Task Force for the Management of Arterial Hypertension of the European Society of Hypertension (ESH) and of the European Society of Cardiology (ESC). J Hypertens 2007, 25:1105-1187.

10. Reboldi G, Gentile G, Angeli F, Verdecchia P: Optimal therapy in hypertensive subjects with diabetes mellitus. Curr Atheroscler Rep 2011, 13:176-185.

11. Mogensen CE: Systemic blood pressure and glomerular leakage with particular reference to diabetes and hypertension. J Intern Med 1994, 235:297-316.

12. Elliott WJ, Meyer PM: Incident diabetes in clinical trials of antihypertensive drugs: a network meta-analysis. Lancet 2007, 369:201-207.

13. Kitamura N, Takahashi Y, Yamadate S, Asai S: Angiotensin II receptor blockers decreased blood glucose levels: a longitudinal survey using data from electronic medical records. Cardiovasc Diabetol 2007, 6:26.

14. Ogihara T, Nakao K, Fukui T, Fukiyama K, Ueshima K, Oba K, Sato T, Saruta T, Candesartan Antihypertensive Survival Evaluation in Japan Trial Group: Effects of candesartan compared with amlodipine in hypertensive patients with high cardiovascular risks: candesartan antihypertensive survival evaluation in Japan trial. Hypertension 2008, 51:393-398.

15. Julius S, Kjeldsen SE, Weber M, Brunner HR, Ekman S, Hansson L, Hua T, Laragh J, Mclnnes GT, Mitchell L, Plat F, Schork A, Smith B, Zanchetti A, VALUE trial group: Outcomes in hypertensive patients at high cardiovascular risk treated with regimens based on valsartan or amlodipine: the VALUE randomised trial. Lancet 2004, 363:2022-2231.

16. Julius S, Weber MA, Kjeldsen SE, McInnes GT, Zanchetti A, Brunner HR, Laragh J, Schork MA, Hua TA, Amerena J, Balazovjech I, Cassel G, Herczeg B, Koylan N, Magometschnigg D, Majahalme S, Martinez F, Oigman W, Seabra Gomes R, Zhu JR: The Valsartan Antihypertensive Long-Term Use Evaluation (VALUE) trial: outcomes in patients receiving monotherapy. Hypertension 2006, 48:385-391.

17. Takahashi Y, Nishida Y, Asai S: Utilization of health care databases for pharmacoepidemiology. Eur J Clin Pharmacol 2011, 68:123-129.

18. Austin PC, Chiu M, Ko DT, Goeree R, Tu JV: Propensity Score Matching for Estimating Treatment Effects. In Analysis of Observational Health Care Dato Using SAS. Edited by Faries DE, Leon AC, Haro JM, Obenchain RL. Cary: SAS Press; 2010:51-84.

19. Takahashi Y, Nishida Y, Nakayama T, Asai S: Adverse effect profile of trichlormethiazide: a retrospective observational study. Cardiovasc Diabetol 2011, 10:45.

20. Rosenbaum PR, Rubin DB: The central role of the propensity score in observational studies for causal effects. Biometrika 1983, 70:41-55.

21. Flores CA, Ardiles LG, Aros CA, Muñoz CC, Schneider HO, Ramírez JA, Jerez $\checkmark$, Valderrama MG, Mezzano SA: Valsartan-induced hematocrit changes in renal transplant patients. Transplant Proc 2005, 37:1586-1588.

22. Ersoy A, Dilek K, Usta M, Yavuz M, Güllülü M, Oktay B, Yurtkuran M: Angiotensin-II receptor antagonist losartan reduces microalbuminuria in hypertensive renal transplant recipients. Clin Transplant 2002, 16:202-205.

23. Naeshiro I, Sato K, Chatani F, Sato S: Possible mechanism for the anemia induced by candesartan cilexetil (TCV-116), an angiotensin II receptor antagonist, in rats. Eur J Pharmacol 1998, 354:179-187. 
24. Formica RN Jr, Friedman AL, Lorber MI, Smith JD, Eisen T, Bia MJ: A randomized trial comparing losartan with amlodipine as initial therapy for hypertension in the early post-transplant period. Nephrol Dial Transplant 2006, 21:1389-1394.

25. Kurnik D, Vesterman-Landes J, Bialik M, Katzir I, Lomnicky Y, Halkin H, Loebstein R: Hyperkalemia and renal function during monotherapy and dual renin-angiotensin blockade in the community setting. Clin Ther 2011, 33:456-464.

26. Eriksson JW, Jansson PA, Carlberg B, Hägg A, Kurland L, Svensson MK, Ahlström H, Ström C, Lönn L, Ojbrandt K, Johansson L, Lind L: Hydrochlorothiazide, but not candesartan, aggravates insulin resistance and causes visceral and hepatic fat accumulation: the mechanisms for the diabetes preventing effect of candesartan (MEDICA) Study. Hypertension 2008, 52:1030-1037.

27. Kavgaci H, Sahin A, Onder Ersoz H, Erem C, Ozdemir F: The effects of losartan and fosinopril in hypertensive type 2 diabetic patients. Diabetes Res Clin Pract 2002, 58:19-25.

28. Munger MA: Use of angiotensin receptor blockers in cardiovascular protection: current evidence and future directions. P T 2011, 36:22-40,

29. Marshall TG, Lee RE, Marshall FE: Common angiotensin receptor blockers may directly modulate the immune system via VDR, PPAR and CCR2b. Theor Biol Med Model 2006, 3:1.

30. Yamana A, Arita M, Furuta M, Shimajiri Y, Sanke T: The angiotensin II receptor blocker telmisartan improves insulin resistance and has beneficial effects in hypertensive patients with type 2 diabetes and poor glycemic control. Diabetes Res Clin Pract 2008, 82:127-131.

31. Mori Y, Tanaka T, Matsuura K, Yokoyama J, Utsunomiya K: Influence of telmisartan on insulin response after glucose loading in obese patients with hypertension: ARB trial of hypertension in obese patients with hyperinsulinemia assessed by oral glucose tolerance test (ATHLETE). Adv Ther 2011, 28:698-706.

32. Sinha N, Mishra TK, Singh T, Gupta N: Effect of iron deficiency anemia on hemoglobin A1c levels. Ann Lab Med 2012, 32:17-22.

33. Ford ES, Cowie CC, Li C, Handelsman Y, Bloomgarden ZT: Iron-deficiency anemia, non-iron-deficiency anemia and $\mathrm{HbA1c}$ among adults in the US. J Diabetes 2011, 3:67-73.

34. Nakamura T, Inoue T, Suzuki T, Kawagoe $Y$, Ueda Y, Koide H, Node K: Comparison of renal and vascular protective effects between telmisartan and amlodipine in hypertensive patients with chronic kidney disease with mild renal insufficiency. Hypertens Res 2008, 31:841-850.

35. Nieschlag E: Behre HM: Testosterone: action, deficiency, substitution. 2nd edition. Berlin: Springer; 1998

36. Nishida Y, Takahashi Y, Nakayama T, Soma M, Kitamura N, Asai S: Effect of candesartan monotherapy on lipid metabolism in patients with hypertension: a retrospective longitudinal survey using data from electronic medical records. Cardiovasc Diabetol 2010, 9:38.

37. Nishida Y, Takahashi Y, Nakayama T, Soma M, Asai S: Comparative effect of olmesartan and candesartan on lipid metabolism and renal function in patients with hypertension: a retrospective observational study. Cardiovasc Diabetol 2011, 10:74

38. Kyvelou SM, Vyssoulis GP, Karpanou EA, Adamopoulos DN, Zervoudaki Al Pietri PG, Stefanadis Cl: Effects of antihypertensive treatment with angiotensin II receptor blockers on lipid profile: an open multi-drug comparison trial. Hellenic J Cardiol 2006, 47:21-28.

doi:10.1186/1475-2840-11-53

Cite this article as: Nishida et al:: Comparative effect of angiotensin II type I receptor blockers and calcium channel blockers on laboratory parameters in hypertensive patients with type 2 diabetes. Cardiovascular Diabetology 2012 11:53.

\section{Submit your next manuscript to BioMed Central and take full advantage of:}

- Convenient online submission

- Thorough peer review

- No space constraints or color figure charges

- Immediate publication on acceptance

- Inclusion in PubMed, CAS, Scopus and Google Scholar

- Research which is freely available for redistribution

Submit your manuscript at www.biomedcentral.com/submit
C Biomed Central 